\title{
Characterization of a Schottky Diode Rectenna for Millimeter Wave Power Beaming Using High Power Radiation Sources
}

\author{
A. Etinger, M. Pilossof, B. Litvak, D. Hardon, M. Einat, B. Kapilevich \\ AND Y. PINHASI* \\ Ariel University, Ariel 40700, Israel
}

\begin{abstract}
Two principal elements play a role in a wireless power beaming system: a high power radiation source as the transmitter and a rectifying antenna (rectenna) as an RF to DC converter at the receiving site. A millimeter wave power transmission is analyzed using transmission system and a W-band rectenna based on a low-barrier Schottky diode. A quasi-optical approach is presented here, using free-space Gaussian propagation and optical ABCD matrices for lenses. Experiments are made to estimate the optimal load resistance and power handling capability of a single rectifier. A low power W-band tunable solid-state source delivering $0.4 \mathrm{~W} \mathrm{CW}$ power equipped by the focusing lenses is used to characterize the responsivity of the rectenna. A pulsed power gyrotron is used to identify the diode breakdown point. It was found that the RF-to-DC conversion efficiency corresponding to the optimal load of $200 \Omega$ is about $20.5 \%$ while the maximum DC power converted by the diode with optimal load is about $15 \mathrm{~mW}$ before breakdown.
\end{abstract}

DOI: 10.12693/APhysPolA.131.1280

PACS/topics: 84.40.--x, 88.80.hp, 88.80.ht, 42.25.Bs

\section{Introduction}

Wireless power beaming (WPT) systems consist of a high power electromagnetic radiation source feeding a transmitting antenna illuminating a rectifying antenna at the receiving site [1]. A schematic illustration of a typical WPT system is shown in Fig. 1. In systems operating in millimeter wavelengths, the electromagnetic wave is generated by powerful radiation sources like gyrotrons and free-electron masers [2]. The transmitting antenna is focusing the beam onto a rectifying antenna (rectenna) located at the receiving site [3]. The rectenna is an array of patches connected to rectifying diodes that convert the RF to a DC voltage [4].

The Schottky diodes are widely used in millimeter wave communication systems and radars, mainly as square-law and envelope detectors, mixers and frequency multipliers. Recently, detectors based on low barrier Schottky diodes have demonstrated an outstanding voltage responsivity, up to $1600 \mathrm{mV} / \mathrm{mW}$ at $87.8 \mathrm{GHz}[5]$. As reported in [4], these diodes can also be integrated with a $\mathrm{mm}$ wave rectenna and operated in a large-signal rectifying mode. However, modeling large-signal behavior needs knowledge of non-linear parameters of a diode. Experimental verification is required to characterize the diode in its non-linear operation regime as a millimeter wave rectifier.

The paper describes a $2 \times 2$ patches rectenna with a single half-wave rectifier based on a low-barrier Schottky diode. Experiments were made to determine the optimal DC load resistance corresponding to the maximum RFto-DC conversion efficiency and to estimate the maximal

*corresponding author; e-mail: yosip@ariel.ac.il

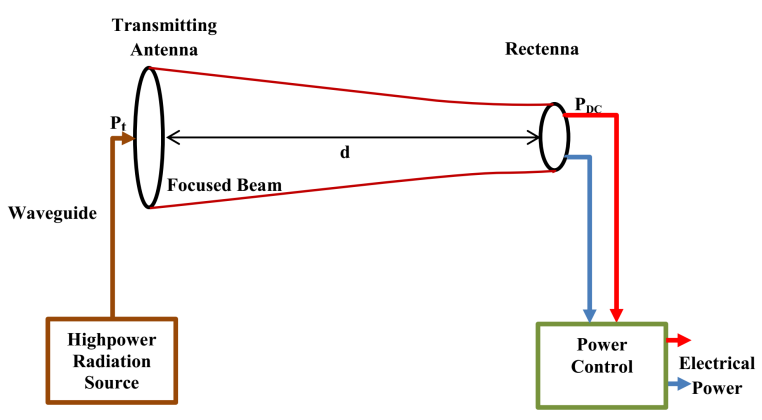

Fig. 1. Schematic illustration of a radiative energy transmission system.

power handling capability for optimal load resistance. The measurements were performed at the W-band, in the vicinity of the $94 \mathrm{GHz}$ atmospheric transmission window. A continuous wave tunable solid-state source with an output power of $0.4 \mathrm{~W}$ has been employed in order to find the optimal load resistance. Then, the millimeter wave pulse generated by W-band gyrotron has been used in order to identify the optimal RF power handling and diode breakdown.

\section{The Schottky diode rectenna element}

The single patch antenna element consists of a rectangular conductor of dimensions $W \times L$ placed on the substrate with metallization process (see Fig. 2). It is excited by a microstrip conductor of the width $t$. The feeding point determined by distance $d$ is the critical parameter responsible for matching the patch to its input port. In our case, the feed network is connected to four patches, resulting in four times impedance reduction at the input port of $2 \times 2$ sub-array.

It is matched with the rectifying circuitry, designed for the input typical impedance of 50-75 $\Omega$. Therefore, the 
impedance of an input port of a single patch should vary within the range 200-300 $\Omega$. It should be noted that the rectifier itself has lower impedance but a reduction of this parameter will require increase of the width of the conductor at the rectifier input. The latter may lead to a packaging problem in building up the final rectenna.

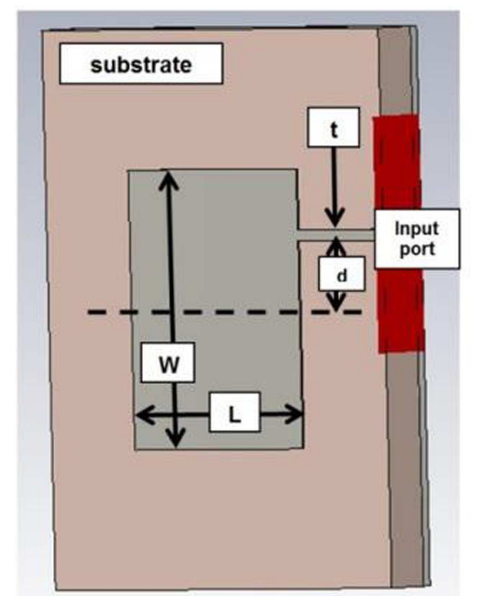

Fig. 2. The single patch antenna element.

Figure 3 shows the calculated reflectance $|S 11|[\mathrm{dB}]$ as a function of frequency for different positions of the feeding point $d$ in $\mathrm{mm}$ when the impedances of the port are $300 \Omega$. Calculations were done using a CST Microwave Studio for the following parameters of the structure: $L=0.87 \mathrm{~mm}, W=1.24 \mathrm{~mm}, t=0.05 \mathrm{~mm}$, height of the substrate, $h=0.254 \mathrm{~mm}$, material of the substrate - Duroid $5880\left(\varepsilon^{\prime}=2.2, \tan \delta=0.001\right)$. It should be noted that in the point of intersection, the input reflectance almost does not depend on the feeding point position $d$.

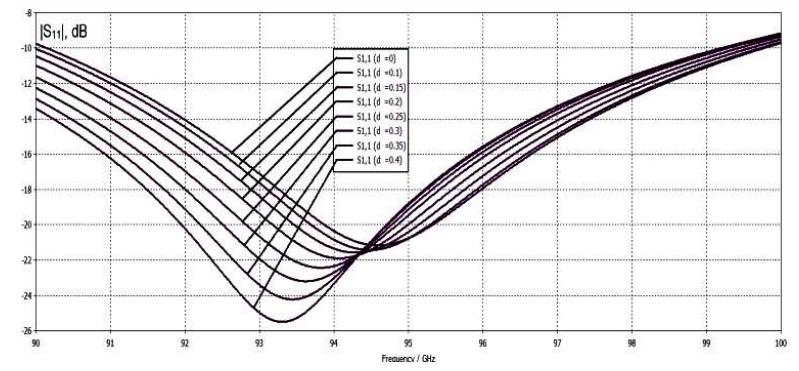

Fig. 3. The calculated reflectance $\left|S_{11}\right|$ in $\mathrm{dB}$ as a function of frequency for different positions of the feeding point $d$ in $\mathrm{mm}$.

The array consisting of $2 \times 2$ patches is shown in Fig. 4 a. The $3 \mathrm{D}$ radiation power gain pattern is shown in Fig. $4 \mathrm{~b}$. At a frequency of $93.5 \mathrm{GHz}$, it is characterized by an absolute gain of $12 \mathrm{~dB}$ leading to $3 \mathrm{~dB}$ beam width of $36.3 \mathrm{deg}$ in the azimuthal plane. The side lobe level is $11.8 \mathrm{~dB}$. The corporate feeding system provides in-phase and equal-amplitude excitations of all 4 patches.

The calculated reflection coefficient $|S 11|[\mathrm{dB}]$ is shown in Fig. 5 for the $75 \Omega$ impedance of the input port. In
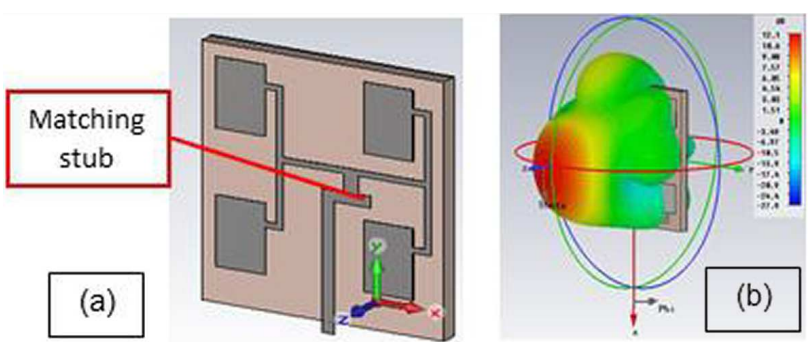

Fig. 4. The array consisting of $2 \times 2$ patches (a) and its $3 \mathrm{D}$ radiation pattern $(\mathrm{b})$.

order to reduce the reflectance and increase the bandwidth, a matching open-circuit stub has been added, as shown in Fig. 4a. By varying the length of this stub, the operating range of $2 \times 2$ patches sub-array can be increased up to $5 \mathrm{GHz}$ for $-25 \mathrm{~dB}$ reflectance level. The latter corresponds to the length stub $=0.2 \mathrm{~mm}$.

In the case of single-diode half-wave rectification, it is preferable to connect the diode in series [5]. Following this recommendation, the rectifier integrated with $2 \times 2$ patches array has been designed, fabricated, and tested. The matching circuitry minimizing the reflection from a low impedance diode has been designed using ADS simulation software. Small-signal parameters of low-barrier Schottky diodes manufactured by IPM RAS, Russia were used in the matching process simulation. The final layout of the rectenna is depicted in Fig. 6.

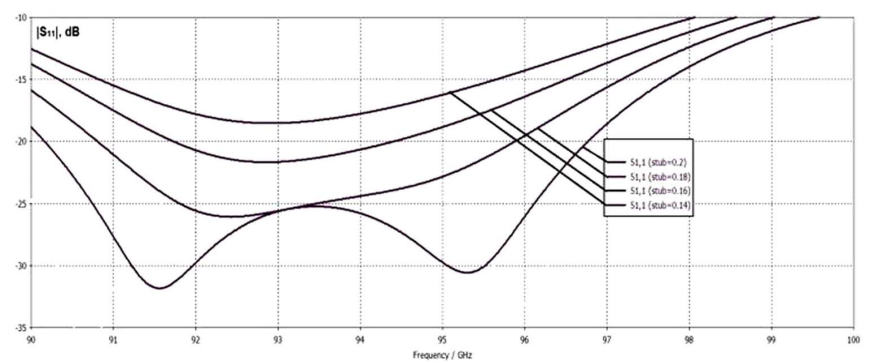

Fig. 5. Effect of variation of the length (in $\mathrm{mm}$ ) of the matching stub on the reflectivity $S 11$.

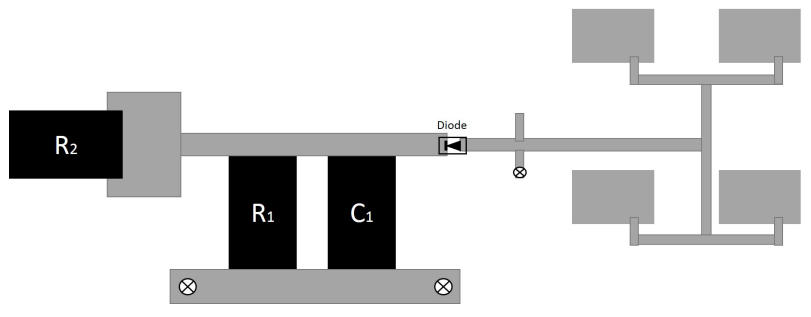

Fig. 6. The layout of $2 \times 2$ patches rectenna with a single half-wave Schottky rectifier.

\section{Quasi optical model for radiative energy transmission}

The transmission system of the energy beaming experiment, shown in Fig. 7 is based on a solid state source 


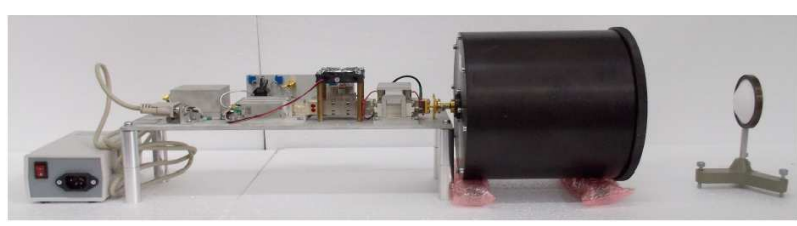

Fig. 7. Experimental setup of wireless power beaming.

producing a power of $400 \mathrm{~mW}$ at $94 \mathrm{GHz}$. The source is connected to a focusing lens antenna, transmitting a beam focused at a distance of $1 \mathrm{~m}$, where a second lens is located, as shown in Fig. 8.

The Hermite-Gaussian TEM modes are a convenient approximate presentation of electromagnetic waves propagating in free-space, and are commonly used in physical optics [6]. Quasi-optical systems operating in the millimeter and sub-millimeter wavelengths are analyzed assuming propagation of the fundamental transverse mode, which is purely Gaussian

$$
\begin{aligned}
& \tilde{E}(r, z)=\tilde{E}_{0}\left(\frac{W_{0}}{W(z)}\right) \exp \left(-\frac{r^{2}}{W^{2}(z)}\right) \\
& \quad \times \mathrm{e}^{-\mathrm{j} \psi(r, z)} \mathrm{e}^{-\mathrm{j} k z} .
\end{aligned}
$$

Here $2 W(z)$ is the typical beam transverse width, where the intensity of the field falls to $1 / e^{2}$ of its maximum intensity attained at the center of the beam $(r=0)$. At the beam waist located at $z=0$, the smallest beam radius $W_{0}$ is obtained. The wavenumber is $k=2 \pi / \lambda$, where $\lambda$ is the radiation wavelength. The Rayleigh distance is $Z_{R}=2 \pi W_{0}^{2} / \lambda$. The typical width of the beam along its propagation axis $z$ is described by

$$
W(z)=W_{0} \sqrt{1+\left(\frac{z}{z_{R}}\right)^{2}} .
$$

The radius of the wave-front curvature as a function of distance $z$ from the beam waist location $(z=0)$ is

$$
R(z)=z\left[1+\left(\frac{z_{R}}{z}\right)^{2}\right] .
$$

The phase term component is

$$
\psi(r, z)=k \frac{r^{2}}{2 R(z)}-\xi(z)
$$

where the term $\xi(z)$ represents the phase slippage of the mode. For the fundamental Gaussian mode

$$
\xi(z)=\arctan \left(\frac{z}{z_{R}}\right) .
$$

In optical Gaussian beam analysis, it is convenient to define a complex beam parameter [7], which includes both the typical beam width $W(z)$ and the wave-front curvature radius $R(z)$ :

$$
\frac{1}{q}=\frac{1}{R(z)}-\mathrm{j} \frac{\lambda}{\pi W^{2}(z)} .
$$

This parameter will be used later in the calculation of the beam spot dimensions on the rectenna. The power intensity along the propagation axis $z$ is given by

$$
\begin{aligned}
& I(r, z)=\frac{1}{2 \eta_{0}}|\tilde{E}(r, z)|^{2}=\frac{\left|\tilde{E}_{0}\right|^{2}}{2 \eta_{0}}\left(\frac{W_{0}}{W(z)}\right)^{2} \\
& \quad \times \mathrm{e}^{-\frac{2 r^{2}}{W^{2}(z)}},
\end{aligned}
$$

where $\eta_{0}=\sqrt{\mu_{0} / \varepsilon_{0}}$ is the impedance of the free-space. The transverse profile of the intensity is Gaussian. The total power carried by the beam is calculated via the integration

$$
P_{\text {tot }}=\int_{0}^{\infty} \int_{0}^{2 \pi} I(r, z) r \mathrm{~d} r \mathrm{~d} \theta=\frac{\pi W_{0}^{2}}{2} \frac{\left|\tilde{E}_{0}\right|^{2}}{2 \eta_{0}} .
$$

Using the last equation, we substitute $\left|\tilde{E}_{0}\right|^{2}$ into Eq. (7), resulting in

$$
I(r, z)=\frac{2 P_{\mathrm{tot}}}{\pi W^{2}(z)} \mathrm{e}^{-\frac{2 r^{2}}{W^{2}(z)}} .
$$

It is convenient to express the effective area of the mode as the ratio between the total power $P_{\text {tot }}$ and the maximum intensity obtained $I(0, Z)$ at $r=0$ :

$$
A_{E M}(z)=P_{\text {tot }} / I(0, z)=\pi W^{2}(z) / 2 .
$$

In our case, a focused Gaussian beam is transmitted by a lens antenna with a typical width of $2 W=108 \mathrm{~mm}$. Using Eq. (2), the width of the waist is found to be $2 W_{01}=40 \mathrm{~mm}$ at a distance of $1 \mathrm{~m}$, where the power intensity at the center of the mode is calculated to be $I_{1}(0,0)=63.66 \mathrm{~mW} / \mathrm{cm}^{2}$.

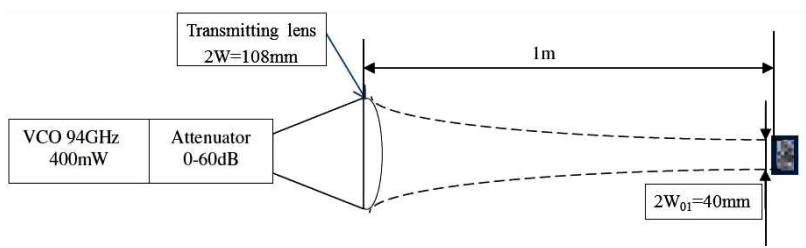

Fig. 8. Propagation of the Gaussian beam from the transmitter lens antenna.

This intensity was found to be insufficient for attaining the optimal RF to DC conversion efficiency. Therefore it was necessary to add a focusing lens in front of the rectenna as shown in Fig. 3, to further reduce the beam spot area, thus increasing the power intensity obtained at the rectenna plane. We employed the ABCD optical matrix to characterize the beam at the focal plane [7]. The complex parameter $q_{2}$ of the beam at the output of an optical system is given in terms of the beam parameter $q_{1}$ at its input according to

$$
q_{2}=\frac{A q_{1}+B}{C q_{1}+D} .
$$

In our case, the optical system consists of a focusing lens with a focal length $f$ and additional free-space propagation to a distance $d$ from the lens where the rectenna will be located. The resulting optical matrix is

$$
\left[\begin{array}{ll}
1 & d \\
0 & 1
\end{array}\right] \cdot\left[\begin{array}{cc}
1 & 0 \\
-\frac{1}{f} & 1
\end{array}\right]=\left[\begin{array}{cc}
1-\frac{d}{f} & d \\
-\frac{1}{f} & 1
\end{array}\right] .
$$


The beam parameter at the lens is $q_{1}=\mathrm{j} \pi W_{01}^{2} / \lambda$ (as of a waist). It is required to focus the beam to a second waist located at a distance $d$ from the lens, as shown in Fig. 8. Thus $q_{2}=\mathrm{j} \pi W_{02}^{2} / \lambda$. Using (10) and (11), we obtained the ratio

$$
\frac{W_{02}}{W_{01}}=\sqrt{1-\frac{d}{f}} .
$$

The second focusing lens is chosen to be with a focal length of $f=38.4 \mathrm{~mm}$. The rectenna is placed at a distance $d=36 \mathrm{~mm}$ from the lens, where according to (12) a typical beam width of $2 W_{02}=10 \mathrm{~mm}$ is obtained. The power intensity at the center of the beam illuminating the rectenna is resulted to be $I_{2}=1 \mathrm{~W} / \mathrm{cm}^{2}$, while the effective waist area is $A_{E M}=\pi W_{02}^{2} / 2=0.39 \mathrm{~cm}^{2}$.

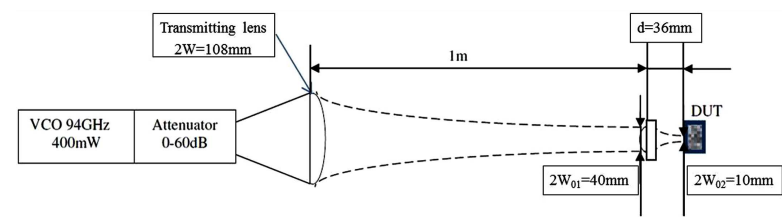

Fig. 9. Focusing a Gaussian beam onto a rectenna using a second lens.

\section{Optimal load resistance}

A tunable continuous wave solid-state W-band source capable of $0.4 \mathrm{~W}$ output power is used to characterize the rectenna. The $2 \times 2$ sub-array rectenna is placed behind the focusing lens of $6 \mathrm{~cm}$ diameter in a position of the beam spot, having a minimum diameter of approximately $1 \mathrm{~cm}$ as shown in Fig. 10. The other focusing lens (diameter $200 \mathrm{~mm}$ ) is a part of the source. The distance between the transmitting antenna and the receiving one is $1 \mathrm{~m}$. The graph of Fig. 11 shows the measured DC power as a function of the load resistances. The maximum output power is $2.7 \mathrm{~mW}$ for a load of $200 \Omega$. The latter corresponds to the $15 \%$ of RF-to-DC conversion efficiency. This estimation was done using the technique reported in $[4,8]$.

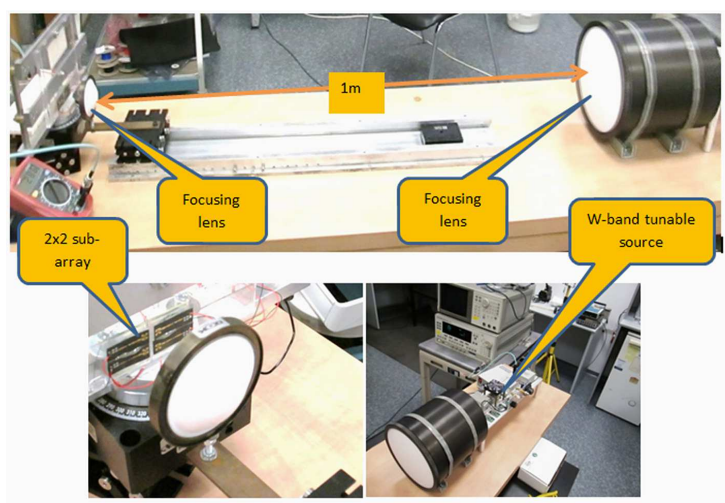

Fig. 10. Experimental setup used for rectenna characterization in the $\mathrm{W}$-band, using a $0.4 \mathrm{~W}$ solid state tunable source.

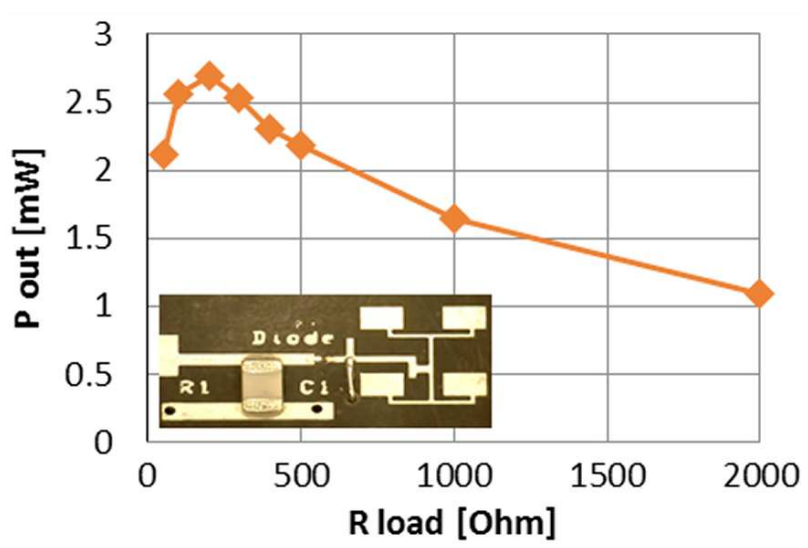

Fig. 11. The measured DC power as a function of the load resistances.

The output power of the source used in previous experiments is not enough for observing the power handling limits. Therefore, a compact medium pulse power W-band gyrotron was also employed.

The gyrotron operates at a frequency of $95 \mathrm{GHz}$ and generates $5 \mathrm{~kW}$ (Fig. 12). It has a pulsed solenoid that produces $3.6 \mathrm{~T}$ of magnetic field. The gyrotron output pulse duration is $15 \mu \mathrm{s}$ [9]. The output mode of the gyrotron is TE02 [10]. The gyrotron output is coupled to a mode converter that converts the radiation first from TE02 to TE01 circular modes, and then to TE10 rectangular mode, coupled into a standard WR10 waveguide. The power is attenuated with a variable attenuator. The maximal power after the attenuation, delivered for illuminating the rectenna was gradually increased up to $\approx 200 \mathrm{~W}$.

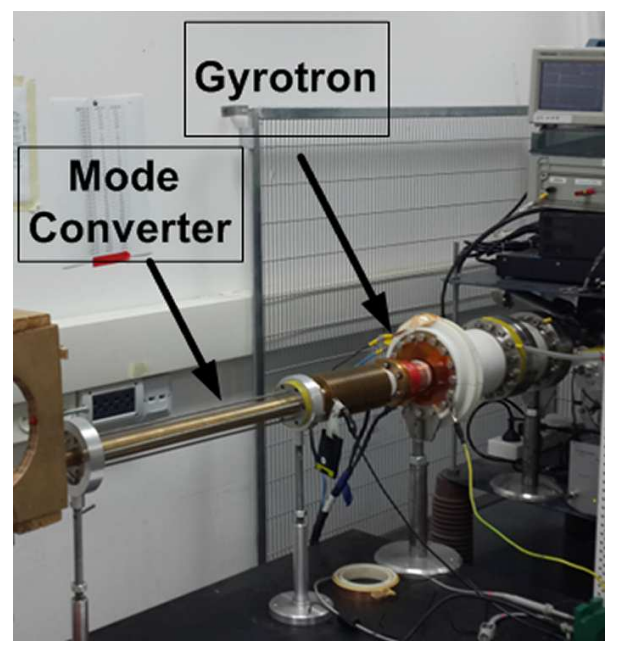

Fig. 12. Medium power (5 kW) W-band gyrotron.

The focusing lens transmitting antenna is now being connected to the gyrotron as shown in Fig. 13, illuminating the rectenna. The output of the gyrotron is coupled to a mode converter that transforms the radiation from TE02 to TE01 of the circular waveguide, and then to TE10 of the rectangular mode with a standard WR10 
waveguide [9]. A radiation power of $5 \mathrm{~kW}$ at the frequency of $95 \mathrm{GHz}$ is generated, and the peak power after attenuation delivered for illuminating the rectenna during $15 \mu \mathrm{s}$ pulse is about $200 \mathrm{~W}$. Figure 14 shows the measured DC power at the rectenna's output as a function of the RF power at the input of the lens antenna. The maximum converted DC power is about $15 \mathrm{~mW}$ before breakdown. It corresponds to an $8.5 \mathrm{~mA}$ current through the diode (for $200 \Omega$ resistance of the load) much more than the $3 \mathrm{~mA}$ allowable current. So we can say that the breakdown is caused by thermal destroying of the diode due to a current overload.

The RF power delivered to the rectenna is reduced due to the following factors:

1. Efficiency of the horn exciting the lens: $E_{\text {horn }}=0.5$;

2. Efficiency of the overall focusing system: $E_{\text {focus }}=$ 0.6 ;

3. Rectifying circuitry mismatching: $E_{\text {rect }}=0.5$;

4. Single-wave rectification efficiency: $E_{\text {single }}=0.3$.

According to Fig. 11, the RF power before breakdown is $160 \mathrm{~W}$ at the input of the lens antenna. The actual $\mathrm{RF}$ power delivered to the rectenna for $\mathrm{DC}$ conversion is $P_{r f}=P_{\text {source }} \times E_{\text {ill }} \times E_{r f} \times E_{\text {rect }}=160 \times 0.5 \times 0.6 \times$ $0.5 \times 0.3=7.2 \mathrm{~W}$.

The effective area of a $2 \times 2$ elements rectenna having gain of $G=12 \mathrm{dBi}$ is equal to $A_{\text {eff }}=\lambda 2 G / 4 \pi=$ $0.128 \mathrm{~cm}^{2}$ for $\lambda=3.2 \mathrm{~mm}$. The diameter of the beam spot illuminating the rectenna is $4 \mathrm{~cm}$ resulting in the actual power delivered to a $2 \times 2$ elements rectenna $7.2 \mathrm{~W} \times 0.128 / \pi \times 22=73 \mathrm{~mW}$. The resulted $\mathrm{RF}$-to-DC conversion efficiency is $15 \mathrm{~mW} / 73 \mathrm{~mW}=20.5 \%$. This is slightly more than the similar result obtained in Sect. 4 .

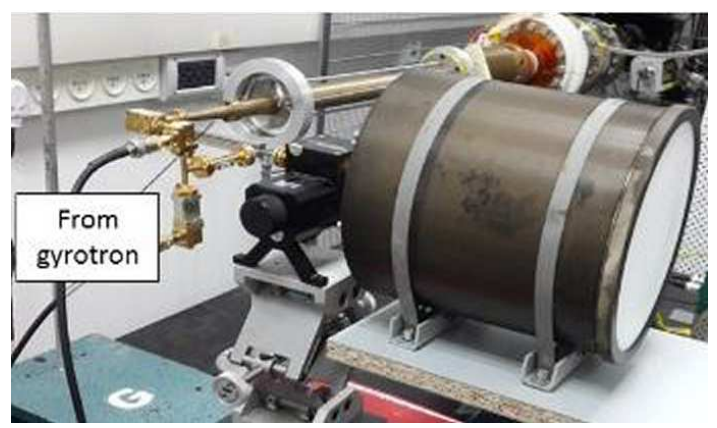

Fig. 13. The experimental setup with the W-band gyrotron.

\section{Conclusion}

The paper presents theoretical and experimental study of radiative energy beaming in millimeter wavelengths. Quasi-optical approach is employed for the analysis of radiation intensity received at the rectifying antenna (rectenna), which converts the millimeter wave radiation to a DC voltage. Gaussian beam analysis, which is commonly used in optical wavelengths, is shown to be applicable also here, while using the fundamental transverse TEM mode. The ABCD matrix is used for the analysis of focusing lenses.

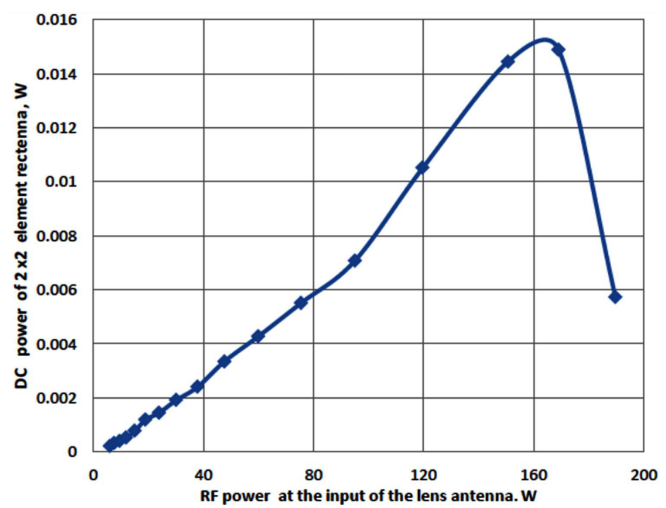

Fig. 14. The measured DC power at the rectenna's output as a function of the RF power at the input of lens antenna.

The $2 \times 2$-patches rectenna operating in $\mathrm{W}$-band has been designed and tested in the non-linear regime of operation. It was demonstrated that the low-barrier Schottky diode may serve as a rectifier in $\mathrm{mm}$ wave rectennas being able to provide up to $15 \mathrm{~mW}$ of DC power per diode at the W-band. Power beaming experiments with a continuous wave solid-state source and a pulsed power gyrotron high demonstrate RF-to-DC conversion efficiency $15-20 \%$ at the W-band.

\section{Acknowledgments}

Authors wish to thank V. Shashkin for his fruitful discussions.

\section{References}

[1] W.C. Brown, IEEE Trans. Microw. Theory MTT32, 1230 (1984).

[2] Y. Pinhasi, I.M. Yakover, A. Eichenbaum, A. Gover, IEEE Trans. Plasma Sci. 24, 1050 (1996).

[3] E. Danieli, A. Abramovich, Y. Pinhasi, IET Microwaves Antennas Propagat. 9, 1167 (2015).

[4] B. Kapilevich, V. Shashkin, B. Litvak, G. Yemini, A. Etinger, D. Hardon, Y. Pinhasi, IEEE Microwave Wireless Compon. Lett. 26, 637 (2016).

[5] M. Hoefle, K. Haehnsen, I. Oprea, O. Cojocari, A. Penirschke, R. Jakoby, J. Infrared Milli Terahertz Waves 35, 891 (2014).

[6] H. Kogelnik, T. Li, Proc. IEEE 54, 1312 (1966).

[7] A. Yariv, Quantum Electronics, Wiley, 3rd ed., 1989.

[8] M. Pinuela, P.D. Mitcheson, S. Lucyszyn, Proc. Power MEMS, 41 (2010).

[9] M. Pilossof, M. Einat, Rev. Sci. Instrum. 86, 016113 (2015).

[10] M. Einat, M. Pilossof, R. Ben-Moshe, H. Hirshbein, D. Borodin, Phys. Rev. Lett. 109, 185101 (2012). 